\title{
Adsorption and equilibrium studies of phenol and para-nitrophenol by magnetic activated carbon synthesised from cauliflower waste
}

\author{
Nidhi Yadav, Dhruv Narayan Maddheshiaya, Shalu Rawat, Jiwan Singh ${ }^{\dagger}$ \\ Laboratory of Environmental Nanotechnology and Bioremediation, Babasaheb Bhimrao Ambedkar University, Lucknow-226025, India
}

\begin{abstract}
In this study, waste cauliflower leaves were used for adsorbent preparation. The waste cauliflower leaves were converted into activated carbon by pyrolysis at two different temperatures $250^{\circ} \mathrm{C}$ and $500^{\circ} \mathrm{C}$ with magnetic property. The prepared adsorbents were denoted as CAC-250 and CAC-500 and characterized by the use of scanning electron microscope (SEM), energy dispersive spectroscopy (EDS), Fourier transform infrared spectroscopy (FTIR) and X-ray diffraction (XRD). The adsorbents were applied for the removal of phenol and PNP from their aqueous solutions. The adsorption of phenol was found very less by the application CAC-250, whereas by the application of CAC-500 the adsorption of both phenol and PNP was enhanced. The maximum adsorption of phenol was found $99 \%$ and that of PNP was found $\sim 100 \%$ using CAC-500, with initial adsorbate concentration $5 \mathrm{mg} / \mathrm{L}$ at $25^{\circ} \mathrm{C}$. The adsorption data was analysed with Langmuir, Freundlich and Temkin isotherm models and different kinetic models that are pseudo first order, pseudo second order, Elovich, intraparticle and pore diffusion model.
\end{abstract}

Keywords: Adsorption, Cauliflower leaves, Isotherm, Phenol, PNP, Proximate analysis

\section{Introduction}

Increasing urbanization, population, and industrial growth have led to increased degradation of the environment through the discharge of various pollutants to the environment. The contamination of water with organic pollutants such as phenols increases the scarcity of drinking water [1]. A continuous release of phenols from petrochemical industries, coal conversion, and other phenol-releasing industries result in surface water and groundwater contamination [2]. Phenolic compounds are considered toxic hazards to the environment due to their low biodegradation rate and they get bioaccumulated in nature that has deleterious effects, and impacts on the environment [3]. Phenol imparts bad taste and odor to the water [4]. It also creates an oxygen demand in water bodies. Phenol is a non-polar organic molecule that less soluble in water, whereas PNP is a water-soluble solid [5]. Both are slightly acidic in an aqueous solution. According to the US Environmental Protection Agency (USEPA), PNP is among the most toxic, non-biodegradable, and bioaccumulative chemical compounds [6] and phenol is also categorized by USEPA as an organic pollutant with priority toxicity due to its higher toxicity, mutagenic

This is an Open Access article distributed under the terms of the Creative Commons Attribution Non-Commercial License (http://creativecommons.org/licenses/by-nc/3.0/) which permits unrestricted non-commercial use, distribution, and reproduction in any medium, provided the original work is properly cited.

Copyright (C) 2020 Korean Society of Environmental Engineers and carcinogenic properties [7]. Code of Federal has regulated the maximum discharge of PNP $576 \mu \mathrm{g} / \mathrm{L}$ per day to control its discharge in water bodies [5] Phenol and PNP pose serious harm to the organisms at very low concentrations. The low solubility of phenol in water means that phenol and its derivatives have a half-life in the range of 2 and 72 [8]. They have high stability and carcinogenic nature and are reported to cause considerable damage and risk to human health. Considering these hazardous impacts of phenols, US Environmental Protection Agency gives the permissible limit in wastewater as less than $1 \mathrm{mg} / \mathrm{L}$ [1] p-nitrophenol (PNP) is a common intermediate chemical compound in the pharmaceutical and pesticide industry [9]. PNP is also produced in many chemical processes like petrochemical, plastic, paint, pulp, paper mill processes, and oil refineries [10]. The molecular structure of phenol includes a benzene ring containing a hydroxyl group; it is a planar molecule. It is a representative of the hazardous aromatic compound range with low solubility in water [11]. Hence removal of these organic pollutants from the effluent is necessary before discharging it into a water body. During chlorination of water containing phenols, chlorinated phenols are formed which have organoleptic properties.

Various methods have been used for the removal of phenol and its derivatives from water, including adsorption [12-13], mem-

Received June 8, 2019 Accepted October 16, 2019

${ }^{\dagger}$ Corresponding author

Email: jiwansingh95@gmail.com

Tel: $+91-522-2995606$ 
brane filtration [14-15], bio-electrochemical treatment [16], electrochemical oxidation [17], ion exchange [18], solvent extraction [19] and fenton degradation [20]. Along with these methods, some detrimental techniques like incineration, ozonation are also applied for the treatment of phenolic contamination [21]. Along with biodegradation, adsorption is one of the primary water treatment processes employed for the purification of water because of the ease of operation, suitability, design simplicity, high removal efficiency, and wide area of application [22]. Water purification by means of adsorption is an attractive approach as it is a flexible technique that can be applied to a range of contaminants and at varying operating conditions without impacting on removal efficiency. A variety of adsorbents are used for the removal of phenol from water, and activated carbon (AC) is the most widely used adsorbent [21]. The cost of the adsorption process is mainly related to selection of the adsorbent. Although AC is highly efficient in removing organic and inorganic contaminants, the application of AC is limited due to its prohibitive cost and the time involved in preparation and regeneration of the adsorbent.

Preparation of an adsorbent with high removal efficiency and with low development and operational cost is necessary. Recent research has focused on the preparation of low-cost adsorbents to reduce the operating costs of adsorption. Recently several studies have been published on low cost adsorbents with high adsorption potential, such as natural zeolite [23], treated waste newspaper [24], canola residue [25], olive mill waste [26], and corn cob residue synthesized hydrochar [27]. Many researchers have proposed utilization of agricultural waste as a raw material for adsorption, as agricultural waste is reported to have high adsorption potential with low cost and high availability [28].

The aim of the present study is to synthesize a low-cost adsorbent using an agriculture waste, namely, waste cauliflower leaves, and investigate its application in the removal of phenol and PNP from aqueous solutions. In developing countries like India, waste cauliflower leaves are a common vegetable waste produced in large quantities, which has high moisture content. Improper disposal of this waste creates a public nuisance. Hence, utilization of this food waste in adsorbent preparation will assist in the management of this solid waste. In the present study, AC was synthesized from waste cauliflower leaves at two different temperatures, $250^{\circ} \mathrm{C}$ and $500^{\circ} \mathrm{C}$, and these adsorbents were applied for the removal of phenol and PNP from water.

\section{Materials and Methods}

\subsection{Chemicals and Reagents}

The raw material (waste cauliflower leaves) used for the preparation of adsorbents was collected from a local market. Ferric chloride, amino anti-pyrene, phenol and p-nitrophenol, and other chemicals were purchased from Sigma-Aldrich and Thermo Fisher Scientific. All the chemicals used in this study were of analytical grade and used directly without any further purification.

\subsection{Adsorbent Preparation}

The collected waste cauliflower leaves were cleaned and rinsed with distilled water several times, dried in a hot air oven for 24 $\mathrm{h}$ at $105^{\circ} \mathrm{C}$ and then ground into fine powder and sieved with 250 microns mesh. The proximate analysis of the leaf powder was carried out. The magnetization of the adsorbent was done following the method of Lunge et al. [29]. A total of $30 \mathrm{~g}$ of leaf powder was mixed with $150 \mathrm{~mL}$ of $10 \% \mathrm{FeCl}_{3}$ solution in a $250 \mathrm{~mL}$ beaker. The material was then stirred for $4 \mathrm{nh}$ on a magnetic stirrer with a hot plate, following which the content was kept overnight to allow for thorough mixing of $\mathrm{FeCl}_{3}$ with the leaf powder. The resultant material was then pyrolyzed in a muffle furnace to prepare magnetic AC. Preparation of the adsorbent was done at two different operating temperatures, $250^{\circ} \mathrm{C}$, and $500^{\circ} \mathrm{C}$. The adsorbent synthesized at $250^{\circ} \mathrm{C}$ was termed cauliflower synthesized AC 250 (CAC-250) and the other synthesized at $500^{\circ} \mathrm{C}$ was termed cauliflower synthesized AC 500 (CAC-500).

\subsection{Characterization of the CAC- 250 and CAC- 500}

The surface morphology of the adsorbents was examined using a scanning electron microscope (JSM 4490, JEOL, Japan) equipped with energy dispersive x-ray spectroscopy. The adsorbents were analyzed to identify surface functional groups using Fourier-transform infrared spectroscopy (FTIR) (NICOLET 6700, Thermo Fisher Scientific U.S.A.) analysis before and after adsorption of phenol and PNP. The powder x-ray diffractometer (PW 3040/60 PanAlytical. Netherlands) was used for the X-ray diffraction (XRD) analysis of the CAC-250 and CAC-500 adsorbents. The samples were scanned in the range of 10 to $80^{\circ}$ at a rate of $2 \% \mathrm{~min}$. The surface charge $\left(\mathrm{pH}_{\mathrm{ZPC}}\right)$ of the adsorbent s CAC-250 and CAC-500 was determined.

\subsection{Batch Adsorption Studies of Phenol and PNP}

The batch adsorption studies of phenol and PNP were performed using both CAC-250 and CAC-500 and their performance was compared. All the experiments were performed in a $250 \mathrm{~mL}$ Erlenmeyer flask. A $50 \mathrm{~mL}$ adsorbate solution with the initial concentration of phenol $5 \mathrm{mg} / \mathrm{L}$ and PNP $10 \mathrm{mg} / \mathrm{L}$ was added to the flask and then a defined dose of adsorbents was added to it. The mixture was then shaken in an orbital incubator shaker with a constant shaking speed 80 RPM over various time intervals. After adsorption, the mixture was centrifuged and the supernatant was analyzed using UV-Visible spectrophotometer for the determination of the final concentration of the phenol and PNP. The PNP has analyzed at the wavelength $317 \mathrm{~nm}$ directly after centrifugation, while for phenol detection a color development method (4-Aminoantipryrene method) was followed and it was analyzed at $510 \mathrm{~nm}$. In order to investigate the optimization of the phenol and PNP adsorption various parameters were changed. The adsorbent doses ranged from 0.5 to $3 \mathrm{~g} / \mathrm{L}$, initial adsorbate concentration varied from $5 \mathrm{mg} / \mathrm{L}$ to $40 \mathrm{mg} / \mathrm{L}$, pH effect was varied in the range of 2 to 12 and temperatures were $25^{\circ} \mathrm{C}, 35^{\circ} \mathrm{C}$, $45^{\circ} \mathrm{C}$ and $55^{\circ} \mathrm{C}$.

The percentage adsorption and adsorption amount ( $q t, \mathrm{mg} / \mathrm{g}$ ) were determined using the equations (1) and (2), respectively:

$$
\% \text { Adsorption }=\frac{\left(C_{0}-C_{t}\right)}{C_{0}} \times 100
$$




$$
q_{t}=\frac{\left(C_{0}-C_{t}\right) \times V}{m}
$$

Where, $\mathrm{C}_{0}$ and $\mathrm{C}_{\mathrm{t}}$ are the concentration of phenol and PNP initially and at a time $(\mathrm{t})$, respectively; $\mathrm{V}$ and $\mathrm{m}$ are the volume of solution in liters and mass of the adsorbent, respectively.

\section{Results and Discussion}

\subsection{Proximate Analysis of Waste Cauliflower Leaves}

Proximate analysis of waste cauliflower leaves was conducted to determine the moisture, ash, volatile organic matter, and fixed carbon content of the raw material. The moisture content of the waste was $70.3 \%$, ash content was $4.6 \%$, volatile content was $3.3 \%$, and total carbon content was $21.8 \%$.

\subsection{Characterization of Synthesized Adsorbent}

\subsubsection{SEM and EDS analysis}

The surface of the synthesized magnetic AC from waste cauliflower leaves was done using scanning electron microscope (SEM). The SEM images of CAC-250 and CAC-500 are presented in Fig. 1(a) and (b) the images show that the particles of the synthesized adsorbents have a rough surface and an irregular shape. The particles of CAC-500 show greater clustering in comparison with CAC-250, that is may be due to the comparatively higher magnetic property of CAC-500 due to it higher iron content.

The elemental composition of the adsorbents is shown in Fig. 1(a) and (b). Both the adsorbents have different elemental composition. In CAC-250, the maximum weight percentage of the carbon is $52.54 \%$ and iron is only $13.41 \%$, whereas, in CAC-500, the maximum weight percentage of iron is $47.17 \%$ and carbon content is $27.27 \%$. This indicates that a greater amount of iron is fixed at a higher temperature which increases the magnetism.

\subsubsection{Functional group analysis}

Fig. 1(c) shows FTIR spectrum of CAC-250 before and after the adsorption of phenol and PNP. The Figure indicates similar peaks in the graphs. The peak $2,915 \mathrm{~cm}^{-1}$ and $2853 \mathrm{~cm}^{-1}$ correspond to the stretching in $-\mathrm{CH}$ bond, the peaks $1,663 \mathrm{~cm}^{-1}, 1,553 \mathrm{~cm}^{-1}$, and $1447 \mathrm{~cm}^{-1}$ correspond to the stretching of $-\mathrm{CO},-\mathrm{NH}$ and $\mathrm{CH}_{3}$ groups, the peak $1,026 \mathrm{~cm}^{-1}$ represents - $\mathrm{CO}$ group, and the peaks at 790 $\mathrm{cm}^{-1}$ and $697 \mathrm{~cm}^{-1}$ correspond to the $-\mathrm{CH}$ stretching. Fig. 1(d) shows the FTIR spectrum of CAC-500 before and after adsorption of phenol and PNP. Some of the peaks are similar indicating the possible
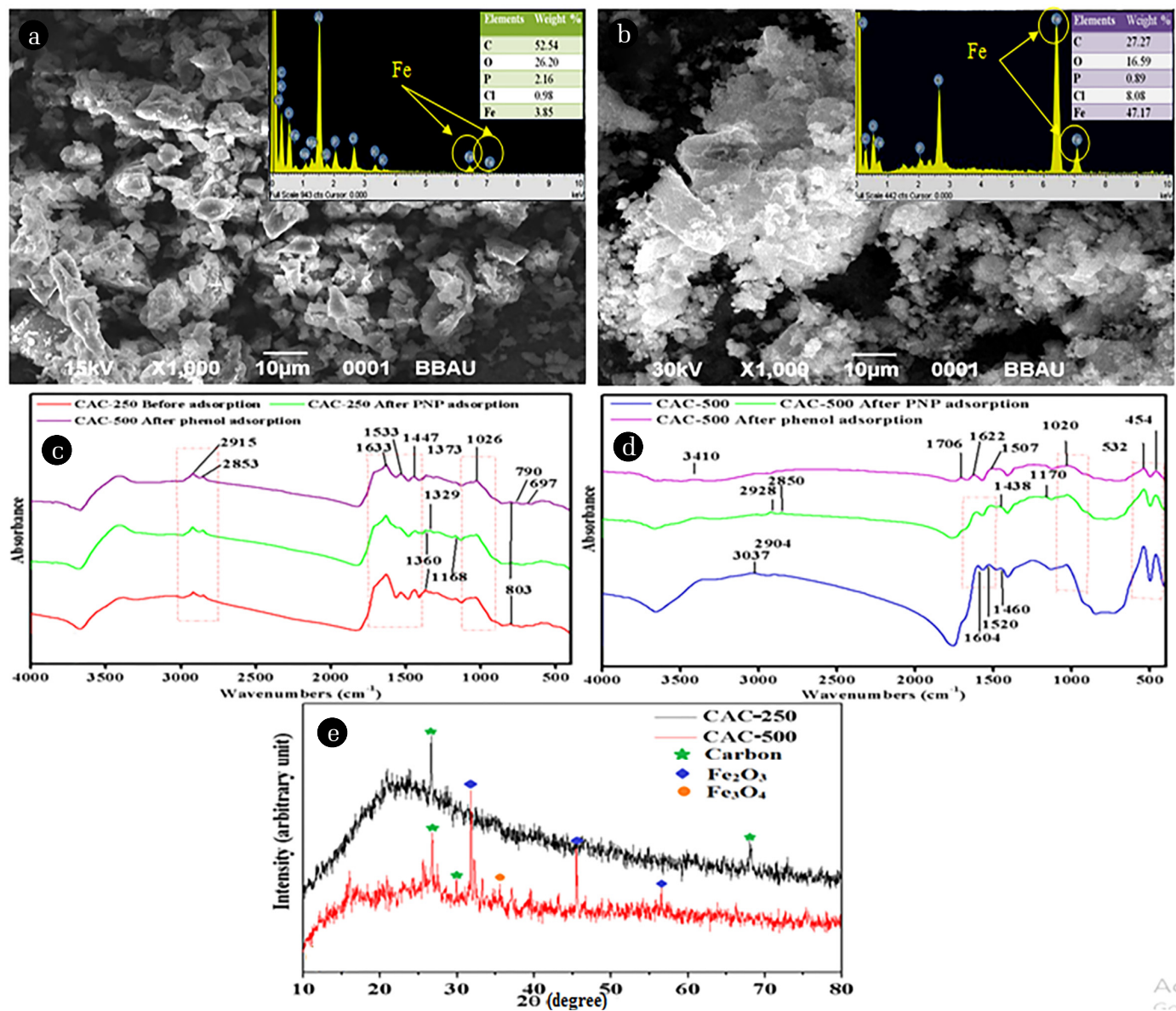

Fig. 1. (a) SEM and EDS image of CAC-250 and (b) CAC-500, (c) FTIR spectra of CAC-250 and (d) CAC-500, respectively and (e) XRD analysis of CAC-250 
presence of the same functional groups, like peak $532 \mathrm{~cm}^{-1}$ and $454 \mathrm{~cm}^{-1}$ that correspond to the presence of iron. These peaks were absent in the spectrum of CAC-250 possibly due to less binding of iron with CAC-250. The peak $3,410 \mathrm{~cm}^{-1}$ present in the spectrum after adsorption of phenol relates to the $-\mathrm{OH}$ stretch in the phenol [30]. The peaks $1,706 \mathrm{~cm}^{-1}$ and $1,170 \mathrm{~cm}^{-1}$ may correspond to the $-\mathrm{C}=\mathrm{O}$ and $-\mathrm{C}-\mathrm{O}$ bond, respectively.

\subsubsection{XRD analysis}

The results of the XRD analysis of both the adsorbents are shown in Fig. 1(e). The result illustrates the CAC-250 spectrum and shows two sharp peaks of carbon with many chaos peaks. The spectrum of CAC-500 shows some sharp peaks along with the carbon peaks, indicating the presence of $\mathrm{Fe}_{2} \mathrm{O}_{3}$ and $\mathrm{Fe}_{3} \mathrm{O}_{4}$ [31] which confirms coating of CAC-500 with $\mathrm{FeCl}_{3}$. The sharp peaks indicate the crystalline nature of the adsorbents.

\subsection{4. $\mathrm{pH}_{Z P C}$}

The $\mathrm{pH}_{\mathrm{ZPC}}$ of the synthesized adsorbents was determined by following the procedure described by Singh et al. [32]. Taken a $50 \mathrm{~mL} 0.01 \mathrm{M}$ solution of $\mathrm{NaCl}$ in different beakers and $\mathrm{pH}$ of the solution was adjusted in the range of $\mathrm{pH} 2$ to 10 with the addition of $0.1 \mathrm{M} \mathrm{HCl}$ and $0.1 \mathrm{M}$
$\mathrm{NaOH}$ solutions. Then $0.2 \mathrm{~g}$ adsorbent was added to the solution and kept for shaking for $48 \mathrm{~h}$ and then the final $\mathrm{pH}$ was measured. A plot between initial $\mathrm{pH}$ and final $\mathrm{pH}$ is shown in the Fig S1. The $\mathrm{pH}_{\mathrm{ZPC}}$ of the CAC-250 was 3.8 while that of CAC-500 was 4.8. The results indicate that the net surface of the adsorbents was positive below the $\mathrm{pH}_{\mathrm{ZPC}}$ value and negative above the $\mathrm{pH}_{\mathrm{ZPC}}$ value.

\subsection{Batch adsorption of Phenol and PNP}

\subsubsection{Effect of adsorbent concentration}

The efficiency of the adsorption process depends on the number of available active adsorption sites. Increasing the adsorbent dose results in an increase in the number of active adsorption sites and increases adsorption of phenol and PNP [33]. In this work, adsorption of phenol and PNP was performed with different dosages of CAC-250 and CAC-500 in the range of $0.5 \mathrm{~g} / \mathrm{L}$ to $3.0 \mathrm{~g} / \mathrm{L}$ at different time interval upto $300 \mathrm{~min}$, with an initial concentration of phenol of $5 \mathrm{mg} / \mathrm{L}$ and $\mathrm{PNP}$ of $10 \mathrm{mg} / \mathrm{L}$ at $25^{\circ} \mathrm{C}$ temperature.

As shown in Fig. 2(a), the maximum adsorption of phenol was found to be $28 \%$ and then the adsorption decreased with increasing dose of CAC-250, an increase in adsorbent concentration may cause adsorbent particles to get agglomerated that enhances the particle
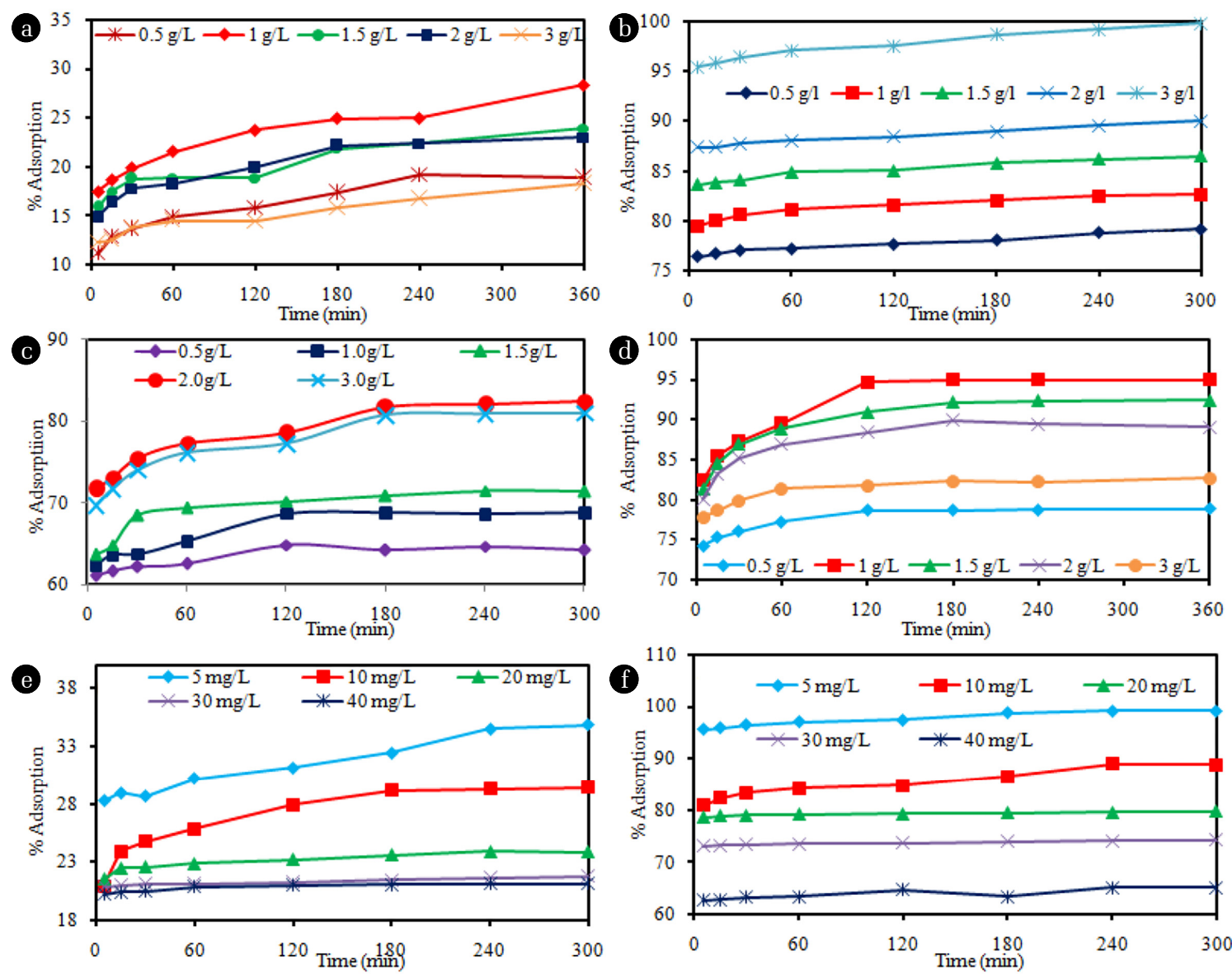

Fig. 2. Effect of adsorbent dose on phenol adsorption using (a) CAC-250 and (b) CAC-500, respectively. Effect of adsorbent dose on PNP adsorption using (c) CAC-250 and (d) CAC-500, respectively, effect of initial concentration of phenol on adsorption by application of (e) CAC-250 and $(\mathrm{f})$ SCAC-500, respectively, effect of initial concentration of PNP on adsorption by application of (d) CAC-250 and (e) CAC-500, respectively. 
size and reduce their surface area which causes a decline in adsorption [34]. Wherever, an opposite trend was observed with CAC-500 the maximum adsorption of phenol was obtained at $3 \mathrm{~g} / \mathrm{L}$ (about 99.76\%) as the Fig. 2(b) shows. Adsorption of PNP was increased with an increase in the dose of both CAC-250 and CAC-500 (Fig. 2(c) and (d)). Maximum adsorption of PNP (95.64\%) was found at $1 \mathrm{~g} / \mathrm{L}$ dose of CAC-500. On increasing the dose up to $3 \mathrm{~g} / \mathrm{L}$, the adsorption of PNP was a decreased significantly from $95.64 \%$ to $82.72 \%$. The adsorption of both the adsorbates using CAC-250 ad CAC-500 increased with increase contact time.

\subsubsection{Effect of initial concentration of phenol and PNP and effect of contact time}

To study the effect of initial phenol and PNP concentrations on the adsorption process, a range of concentrations from $5 \mathrm{mg} / \mathrm{L}$ to $40 \mathrm{mg} / \mathrm{L}$ of phenol and PNP was studied. As shown in Fig. 2(e)-(f) and Fig. 3(a)-(b), the adsorption percentage of phenol and PNP decreases with the increasing initial concentration of both the pollutants by the adsorbents CAC-250 and CAC-500. Maximum adsorption of phenol (99.12\% by CAC-500; $34.80 \%$ by CAC-500) and PNP (100\% by CAC-500; $83.74 \%$ by CAC-50) was found when initial concentrations were $5 \mathrm{mg} / \mathrm{L}$; however, minimum adsorption was found at initial concentration of $40 \mathrm{mg} / \mathrm{L}$. Adsorption $q_{t}$ value increased with an increase in the initial concentration of phenol and PNP, from 1.65 to $8.68 \mathrm{mg} / \mathrm{g}$ and from 4.97 to $33.36 \mathrm{mg} / \mathrm{g}$ for phenol and PNP, respectively, using CAC-500. At low concentrations of phenol and PNP, there were fewer adsorbate molecules. On increasing the concentration of phenol and PNP, the number of adsorbate molecules increased while the adsorption sites remain the same due to the saturation effect, the increase in initial pollutant concentration lead to a decrease in adsorption rate [35].

The variation in the adsorption of phenol and PNP at different contact times was studied and the results are shown in Fig. 2(a)-(f) and Fig. 3(a)-(f). As the figure illustrates, the adsorption of phenol and PNP by application of both the adsorbents was increased with increasing contact time up to $240 \mathrm{~min}$ for phenol and $180 \mathrm{~min}$ for PNP (except for a few cases). Beyond this contact time, the adsorption percentage roughly stabilized, and there was no further significant increase in the adsorption which indicates that the adsorption process reached an equilibrium at 240 and $180 \mathrm{~min}$ for phenol and PNP, respectively.

\subsubsection{Effect of $\mathrm{pH}$ on the adsorption process}

A $\mathrm{pH}$ of the solution is a critical adsorption influencing parameter.
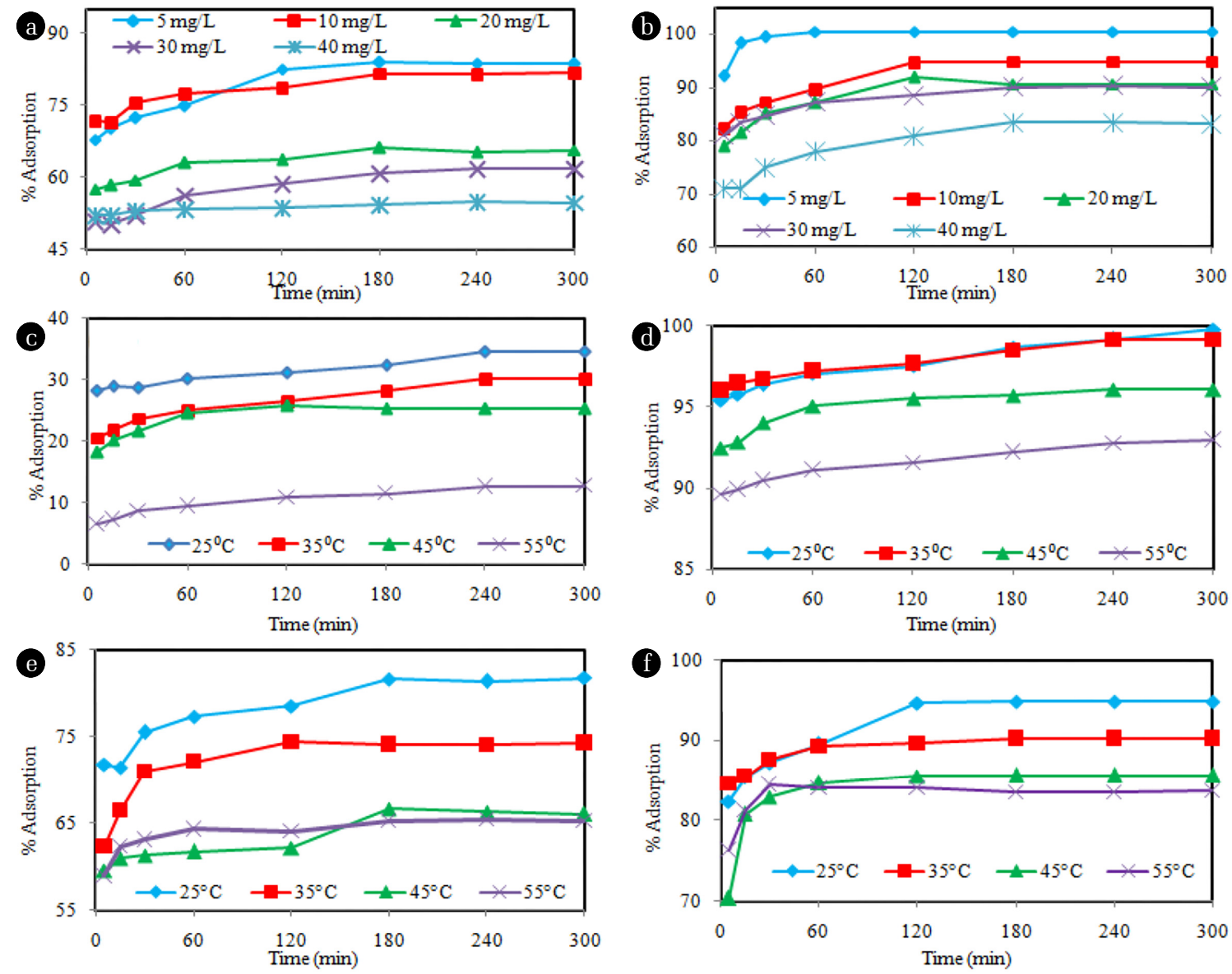

Fig. 3. Effect of initial PNP concentration using (a) CAC-250 and (b) CAC-500, respectively, effect of different temperature on the adsorption of phenol by the application of (c) CAC-250 and (d) CAC-500, respectively, effect of different temperature on the adsorption of PNP by the application of (e) CAC-250 and (f) CAC-500, respectively. 
The solubility of phenol and PNP and charge transfer of the adsorbent is greatly influenced by the concentration of $\mathrm{H}^{+}$ions [36]. To study the effect of $\mathrm{pH}$ on the adsorption of phenol and PNP by CAC-250 and CAC-500, the adsorption was performed at different $\mathrm{pH}$ from $\mathrm{pH} 2$ to 12 as shown in the Fig. 4a the result showed that the adsorption of phenol was increased in alkaline $\mathrm{pH}$ by both CAC-250 and CAC-500 up to $\mathrm{pH} 10$ and then the removal percentage decreased, the effect of $\mathrm{pH}$ on phenol adsorption is more visible by using CAC-500, the maximum adsorption percentage (98.40\%) was found at $\mathrm{pH} 10$ using CAC-500. The molecules of phenol contains a $-\mathrm{OH}$ group of which hydrogen is slightly positively charged and get attracted towards the negatively charged species on increasing the $\mathrm{pH}$ of the solution the adsorbent may carry negative charged that attracted the phenol molecules in the solution that may responsible for high adsorption efficiency of phenol in alkaline $\mathrm{pH}$. Besides electrostatic attraction between the charges of the adsorbent surface and adsorbate molecules the $\mathrm{pH}$ also affects the degree of adsorbate ionization. Phenol is a weak acid that has $\mathrm{pK}_{\mathrm{a}} \sim 9.89$ on a $\mathrm{pH}$ value higher than this the phenol molecules tends to dissociate [37, 38], this dissociation at $\mathrm{pH}$ higher than 10 may result in decrease in the adsorption. The $\mathrm{pK}_{\mathrm{a}}$ value of PNP is reported to be 7.13 [39] that is lower than that of phenol, however, the adsorption of PNP was not much affected by the $\mathrm{pH}$ changed which represents that PNP adsorption was not depend on the electrostatic attraction between ionised PNP species and adsorbent, a similar effect of $\mathrm{pH}$ on PNP adsorption was also reported by [40]. That may be due to the fact that PNP at low $\mathrm{pH}$ remains in the molecular form and get ionised at higher $\mathrm{pH}$ and these ionised species get attracted towards the opposite charges on the adsorbent surface [41].

\subsubsection{Effect of temperature on the adsorption process}

Temperature has a significant effect on adsorption. As shown in Fig. 3(c)-(e), the adsorption of phenol and PNP using both adsorbents CAC-250 and CAC-500 was an exothermic process. The adsorption percentage of phenol and PNP was reduced on increasing temperature; the maximum removal of phenol and PNP was 99.76\% and 94.89\%, respectively, using CAC-500. The adsorption amount of phenol and PNP decreased from 1.65 to $1.54 \mathrm{mg} / \mathrm{g}$ and 4.74 to $4.17 \mathrm{mg} / \mathrm{g}$, respectively, with increasing temperature.

\subsection{Study of Adsorption Isotherms}

\subsubsection{Langmuir adsorption isotherm}

The Langmuir adsorption isotherm considers adsorption of solute in a single layer, uniformly on to an adsorbent surface. The equation of the Langmuir Isotherm can be expressed as given below [42]:

$$
\frac{C_{e}}{q_{e}}=\frac{1}{Q_{0} b}+\frac{C_{e}}{Q_{0}}
$$

Where, $\mathrm{C}_{\mathrm{e}}$ is the equilibrium concentration of phenols and PNP (mg/L) and $\mathrm{q}_{\mathrm{e}}$ is the number of phenols, and PNP adsorbed per gram of adsorbent (mg/g). The Langmuir constant $\mathrm{Q}_{0}$ and $\mathrm{b}$ represent the maximum adsorption capacity of CAC-250 and CAC-500 and rate of adsorption, respectively. Values of $\mathrm{Q}_{0}$ and $\mathrm{b}$ can be determined by the slope and intercept of the $\mathrm{C}_{\mathrm{e}}$ versus $\mathrm{C}_{\mathrm{e}} / \mathrm{q}_{\mathrm{e}}$ plot (Fig. 4(b)). The value of $\mathrm{Q}_{0}$ for CAC-250 was found to be higher for phenol
(15.92 $\mathrm{mg} / \mathrm{g}$ ), while the value of $\mathrm{Q}_{0}$ for CAC-500 was found to be higher for PNP (37.73 mg/g). Values for the Langmuir isotherm parameters are given in Table 1 .

$\mathrm{R}_{\mathrm{L}}$ (the dimensionless constant) is an essential feature of the Langmuir isotherm. It can be expressed as follows:

$$
R_{L}=\frac{1}{\left(1+b C_{0}\right)}
$$

Here, $b$ and $\mathrm{C}_{0}$ are the Langmuir constant and initial adsorbate concentration. The $\mathrm{R}_{\mathrm{L}}$ value represents the favorability of the adsorption process. If the value of $\mathrm{R}_{\mathrm{L}}>1$ it represents unfavorable adsorption, $\mathrm{R}_{\mathrm{L}}=1$ represents a linear isotherm, and $0<\mathrm{R}_{\mathrm{L}}<1$ represents a favorable process of adsorption. The $\mathrm{R}_{\mathrm{L}}$ value for phenol adsorption was found to be 0.44 and 0.05 for CAC-250 and CAC-500, respectively. The values of $\mathrm{R}_{\mathrm{L}}$ for PNP adsorption were found to be 0.10 and 0.02 for CAC-250, and CAC-500, respectively, i.e., between 0 and one, indicates that the adsorption process for both adsorbents is favorable.

\subsubsection{Freundlich Isotherm}

The Freundlich isotherm equation is given below:

$$
\ln q_{e}=\ln K_{F}+\left(\frac{1}{n}\right) \ln C_{e}
$$

Where, $q_{e}$ and $C_{e}$ are the adsorption quantity (mg/g) and concentration of adsorbate $(\mathrm{mg} / \mathrm{L})$ at equilibrium. The Freundlich constants $\mathrm{K}_{\mathrm{F}}$ and $\mathrm{n}$ are the adsorption capacity $\left(\mathrm{mg} / \mathrm{g}(\mathrm{L} / \mathrm{mg})^{1 / \mathrm{n}}\right)$ and adsorption favorability, respectively. Values of both constants can be obtained from the slope and intercept of the plot between $\ln C_{e}$ and $\ln q_{e}$ (Fig. 4(c)), and the values are shown in Table 1. A value of slope $(1 / n)$ between $0-1$ indicates the heterogeneous nature of the adsorbent surface.

\subsubsection{Temkin Isotherm}

The Temkin model assumes a decrease in the adsorption heat of all the molecules in the layer, caused by interaction between the adsorbent and adsorbate. The decrease is linear with coverage and a characteristic feature of the adsorption process is the even distribution of binding energies up to maximum binding energy. The equation for Temkin isotherm is represented below in Eq. (6) [6]:

$$
q_{e}=B_{T} \ln A_{T}+B_{T} \ln C_{e}
$$

Where, $\mathrm{q}_{\mathrm{e}}$ and $\mathrm{C}_{\mathrm{e}}$ are the amounts of phenol and PNP adsorbed on CAC-250 and CAC-500 (mg/g) and the concentration of phenol and PNP (mg/L), respectively. The Temkin isotherm constant is given by $\mathrm{B}_{\mathrm{T}}$, which is the adsorption heat (L/g). The Temkin isotherm equilibrium binding constant is given by $A_{T}$ which is dimensionless. The plots for the Temkin isotherm are shown in Fig. 4(d). The values of $B_{T}$ and $A_{T}$ are represented in Table 1 .

On comparing data of the all three isotherms, the adsorption of phenol is explained well with the Freundlich for CAC-250, however for CAC-500 the values of $\mathrm{R}^{2}$ for Langmuir isotherm and for Freundlich isotherm were comparable, whereas the adsorption of PNP fitted well with Freundlich isotherm. 

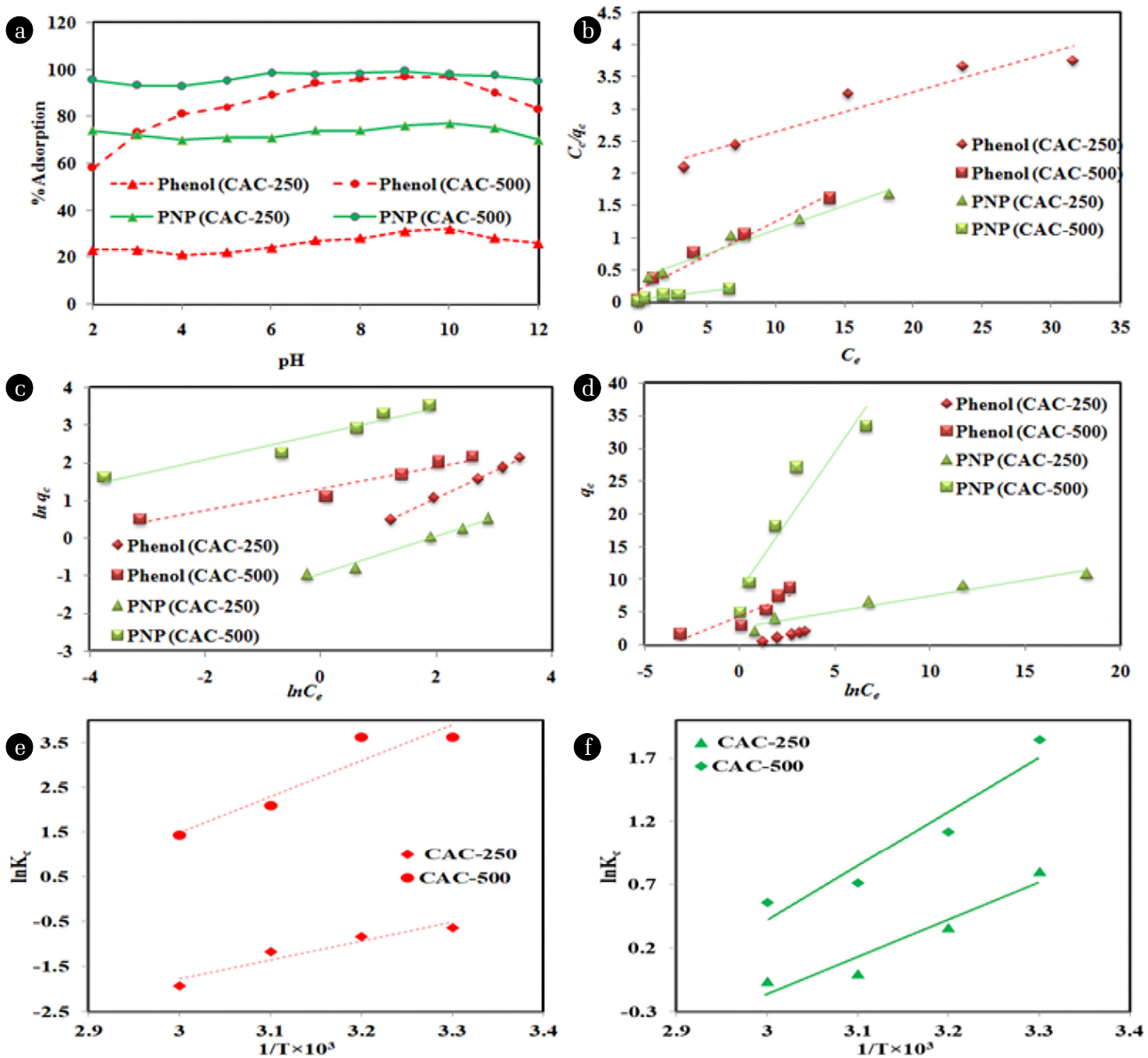

Fig. 4. (a) Effect of $\mathrm{pH}$ on phenol and PNP adsorption, and (b) Plots for Langmuir, (c) Freundlich and (d) Temkin adsorption isotherm, respectively, and Thermodynamics plots for the adsorption of (e) Phenol and (f) PNP, respectively.

Table 1. Values of Langmuir, Freundlich and Temkin Isotherm Parameters for the Adsorption of Phenol and PNP

\begin{tabular}{|c|c|c|c|c|c|}
\hline \multirow{3}{*}{ Isotherms } & & \multicolumn{4}{|c|}{ Parameters } \\
\hline & & \multicolumn{2}{|c|}{ CAC-250 } & \multicolumn{2}{|c|}{ CAC-500 } \\
\hline & & Phenol & PNP & Phenol & PNP \\
\hline \multirow{4}{*}{$\begin{array}{l}\text { Langmuir } \\
\text { Isotherm }\end{array}$} & $\mathrm{Q}_{0}(\mathrm{mg} / \mathrm{g})$ & 15.923 & 13.28 & 9.671 & 37.735 \\
\hline & b (L/mg) & 0.031 & 0.2027 & 0.457 & 0.871 \\
\hline & $\mathrm{R}_{\mathrm{L}}$ & 0.441 & 0.109798 & 0.051 & 0.027 \\
\hline & $\mathrm{R}^{2}$ & 0.916 & 0.9733 & 0.953 & 0.934 \\
\hline \multirow{3}{*}{$\begin{array}{l}\text { Freundlich } \\
\text { Isotherm }\end{array}$} & $\mathrm{K}_{\mathrm{F}}\left(\mathrm{mg} / \mathrm{g}(\mathrm{L} / \mathrm{mg})^{1 / \mathrm{n}}\right)$ & 1.414 & 2.597 & 3.616 & 15.89 \\
\hline & $\mathrm{n}$ & 1.4 & 2.014 & 3.437 & 2.901 \\
\hline & $\mathrm{R}^{2}$ & 0.990 & 0.980 & 0.943 & 0.944 \\
\hline \multirow{3}{*}{$\begin{array}{l}\text { Temkin } \\
\text { Isotherm }\end{array}$} & $\mathrm{A}_{\mathrm{T}}$ & 0.42943 & 2.451486 & 39.36288 & 55.3677 \\
\hline & $\mathrm{B}_{\mathrm{T}}$ & 2.8929 & 2.6972 & 1.1635 & 4.8235 \\
\hline & $\mathrm{R}^{2}$ & 0.9406 & 0.9721 & 0.8024 & 0.8123 \\
\hline
\end{tabular}




\subsection{Kinetics study}

The kinetics for the adsorption process of phenol and PNP on the CAC-250 and CAC-500 was tested by the application of five primary kinetic models that are pseudo first order, pseudo second order, Elovich, intraparticle diffusion and Bangham's pore diffusion model.

The pseudo-first-order kinetics rate expression as given by Hameed and Ahmad [43]:

$$
\log q_{e}-q_{t}=\log q_{e}-\left(\frac{k_{1}}{2.303}\right) t
$$

Here, $q_{e}$ and $q_{t}$ represent the adsorption quantity (mg/g) of phenol and PNP at an adsorption equilibrium state and at a time (t). The value $k_{1}$ represents the adsorption rate constant of phenol and PNP. From the plot of $\log \left(q_{e}-q_{t}\right)$ versus time $(t)$, the value of $k_{1}$ can be calculated (Fig. S2).

The expression for the pseudo-second-order is represented below [44]:

$$
\frac{t}{q_{t}}=\frac{1}{k_{2} q_{e}^{2}}+\frac{1}{q_{e}} t
$$

Where $k_{2}$ is constant ( $\mathrm{g} /(\mathrm{mg} \min )$ ) for the pseudo-second-order and $\mathrm{q}_{\mathrm{e}}$ is the adsorption amount of phenol and PNP, and their values can be evaluated from the slope and intercept of the plot of $t / q t$ versus $t$ (Fig. S3).

The below Eq. (9) shows the Elovich kinetic model:

$$
q_{t}=\frac{1}{\beta \ln (\alpha \beta)}+1 / \beta \ln t
$$

Where, $\mathrm{q}_{\mathrm{t}}$ express the amount of phenol and PNP adsorbed on the adsorbent at $\mathrm{t}$ time (mg/) and $\alpha$ and $\beta$ represents the constant for Elovich kinetic model. $\alpha$ is related with the chemisorptions and $\beta$ is related with the surface coverage extent, values of these constants was obtained by the slope and intercept of the plot between $q_{t}$ and $\operatorname{lnt}$ (Fig. S4).

The intraparticle diffusion model is mainly applied to rate-limiting state adsorption systems. Via intraparticle diffusion, the adsorbate from the bulk state is transferred to the liquid phase [32]. The equation for intraparticle diffusion is represented below [45]:

$$
q_{t}=k_{i} t^{1 / 2}+C
$$

Here, $k_{i}\left(\mathrm{mg} / \mathrm{g} \mathrm{min}{ }^{1 / 2}\right)$ is the rate constant for intraparticle diffusion and the constant $\mathrm{C}$ is related with the boundary layer thickness, a higher value of $\mathrm{C}$ represents the higher boundary layer effect. The application of intraparticle diffusion model is done in three various forms first the straight line passes through the origin which means amount of adsorption at any time, second is when plot qt versus $t^{1 / 2}$ is multi liner which represents the involvement of two or three process in the adsorption and third one which has a straight line that not passes through the origin necessarily (has positive intercept) which indicates that in a short period of time a rapid adsorption has taken place [46]. Intraparticle diffusion is the sole rate-limiting step if the plot of $\mathrm{q}_{\mathrm{t}}$ versus $\mathrm{t}^{1 / 2}$ gives a linear regression that passes through the origin. However, in this study, the linear plots of the phenol and PNP at each concentration do not pass through the origin (Fig. S5); therefore, for the adsorption process of phenol and PNP, intraparticle diffusion is not the only rate limiting step, however, the plot of intraparticle has straight with positive intercept value that shows the rapid adsorption of phenol and PNP in short time period.

The banghman's pore diffusion model was also applied to the adsorption data of phenol and PNP. The equation for the pore diffusion model is given below [3]

$$
\left[\log \left(\frac{C_{0}}{C_{0}-q_{t} \times m}\right)\right]=\log \left(\frac{k_{\text {diff }} \times m}{2.303 \times V}\right)+\alpha \log t
$$

Here, $C_{0}$ denotes the initial phenol and PNP concentration (mg/L), $q_{t}$ denotes the adsorbed amount of phenol and PNP at time $t(\mathrm{mg} / \mathrm{g})$ while, $V$ denotes the volume of the solution treated $(\mathrm{mL})$ and $\mathrm{m}$ denotes the weight of the adsorbent used. $\alpha$ and $k_{\text {diff }}$ are the constants for the pore diffusion model that are evaluated from the slope and intercept of the $\log [\log (\mathrm{Co} / \mathrm{C} 0-\mathrm{qt} \times \mathrm{m})]$ versus $\log \mathrm{t}$ plot (Fig. S6).

The derived values for all the kinetic models are listed in Table S1 and Table S2. The regression coefficient $\left(R^{2}\right)$ of pseudo second order was found maximum that was more than 0.99. It was found that the adsorption of phenol and PNP was best described by the pseudo-second-order kinetics.

\subsection{Thermodynamics}

The value of the thermodynamic parameters, enthalpy ( $\Delta \mathrm{H} ; \mathrm{kJ} / \mathrm{mol})$, entropy $(\Delta S ; \mathrm{kJ} / \mathrm{mol})$ and Gibbs free energy $(\Delta G ; \mathrm{kJ} / \mathrm{mol})$ was estimated by the equation given below [47]:

$$
\begin{gathered}
K_{c}=\frac{q_{e}}{C_{e}} \\
\ln K_{c}=\frac{\Delta S}{R}-\frac{\Delta H}{R T} \\
\Delta G^{\circ}=-R T \ln K_{c}
\end{gathered}
$$

Where, $\mathrm{R}(8.314 \mathrm{~J} / \mathrm{mol} \mathrm{K})$ is gas constant, $q_{e}(\mathrm{mg} / \mathrm{g})$ the adsorbed amount of adsorbate at equilibrium, $C_{e}(\mathrm{mg} / \mathrm{L})$ concentration of phenol and PNP at equilibrium, $K_{c}(\mathrm{~L} / \mathrm{g})$ and $T(\mathrm{~K})$ is absolute temperature. The values of $\Delta H^{\circ}$ and $\Delta S^{\circ}$ can be evaluated by the slope and intercept of $\ln K_{c}$ and $1 / T$ plot as shown in Fig. 4(e) and (f). The values of thermodynamic parameters are shown in Table 3.

The negative value of $\Delta H^{\circ}$ (from -24.48 to $-67.10 \mathrm{~J} / \mathrm{mol}$ ), indicates an exothermic adsorption process and indicates the release of heat during adsorption. The exothermic process mainly represents the physio-sorption or chemisorptions process, physio-sorption is characterized by the value $\Delta H^{\circ}$ lower than $41.86 \mathrm{~kJ} / \mathrm{mol}$ while this value reaches upto $100 \mathrm{~kJ} / \mathrm{mol}$ for a chemisorption processes, in the present study the value of enthalpy is less than $41.86 \mathrm{~kJ} / \mathrm{mol}$ which represents that the adsorption process is physical in nature. 
Table 2. Adsorption Capacity of Different Adsorbent for the Adsorption of Phenol and PNP

\begin{tabular}{|c|c|c|c|c|c|}
\hline \multicolumn{3}{|c|}{ Phenol } & \multicolumn{3}{|c|}{ PNP } \\
\hline Adsorbent & Adsorption capacity & References & Adsorbent & Adsorption capacity & References \\
\hline $\begin{array}{l}\text { Z16 and C16 modified } \\
\text { montmorillonite (C16-Mt) }\end{array}$ & $\begin{array}{l}9.9 \text { and } 13.76 \\
\text { respectively }\end{array}$ & {$[41]$} & Activate carbon fibres & 384.62 & {$[48]$} \\
\hline $\begin{array}{l}\text { Activated carbon prepared } \\
\text { from tobacco residue }\end{array}$ & 17.83 & {$[36]$} & $\begin{array}{l}\text { Olive cake base activated } \\
\text { carbon }\end{array}$ & 1.550 & {$[6]$} \\
\hline $\begin{array}{c}\text { Multiwalled carbon } \\
\text { nanotubes }\end{array}$ & 32.25 & {$[42]$} & $\begin{array}{l}\text { Salycilaldehyde modified } \\
\text { chitosan (CS-SA) }\end{array}$ & 8.5 & {$[49]$} \\
\hline CAC-250 & 15.923 & Present study & CAC-250 & 13.28 & Present study \\
\hline CAC-500 & 9.671 & Present study & CAC-500 & 37.73 & Present study \\
\hline
\end{tabular}

Table 3. Values for thermodynamic parameters for the adsorption of phenol and PNP

\begin{tabular}{|c|c|c|c|c|c|}
\hline \multirow{2}{*}{ Parameters } & \multirow{2}{*}{ Temperature (K) } & \multicolumn{2}{|c|}{ CAC 250} & \multicolumn{2}{|c|}{ CAC 500} \\
\hline & & Phenol & PNP & Phenol & PNP \\
\hline \multirow{4}{*}{$\Delta \mathrm{G}^{\circ}(\mathrm{KJ} / \mathrm{mol})$} & 298 & 35.61 & 22.28 & 56.235 & 30.733 \\
\hline & 308 & 36.81 & 23.03 & 58.125 & 31.765 \\
\hline & 318 & 38.01 & 23.78 & 60.014 & 32.798 \\
\hline & 328 & 39.20 & 24.52 & 61.903 & 33.830 \\
\hline$\Delta \mathrm{H}^{\circ}(\mathrm{J} / \mathrm{mol})$ & & -34.95 & -24.48 & -67.1015 & -35.574 \\
\hline$\Delta \mathrm{S}^{\circ}(\mathrm{J} / \mathrm{mol} \mathrm{K})$ & & -119.63 & -74.86 & -188.936 & -103.252 \\
\hline
\end{tabular}

The negative $\Delta S^{\circ}$ also supports the physio-sorption process of phenol and PNP adsorption. The value of $\Delta G^{\circ}$ is positive for all the adsorption processes and increases with an increase in temperature.

The Table 2 represents the adsorption capacities of different adsorbents for the adsorption of phenol and PNP. The adsorption capacity of adsorbents prepared in the present study is not maximum but it is better from the other reported adsorbents. However, a comparatively easy synthesis with magnetic property and the utilization of waste biomass for adsorbent preparation make its application less costly and effective for the removal of these organic polllutants.

\subsection{Proposed Mechanism for Phenol and PNP Adsorption}

The adsorption of phenol was significantly affected by heat treatment of AC. The adsorption of phenol was much lower with use of CAC-250 in comparison to CAC-500, similar results were also reported by Ibrahim et al. [50] and Mishra et al. [51] who developed activated carbon from sweet lime waste and waste corn husk, respectively, at different temperature and found the activated carbon synthesized at higher temperature more effective in comparison with that synthesized at lower temperature. This may be possibly due to the electrostatic interaction between hydroxides of iron and phenolic $-\mathrm{OH}$. The adsorbent prepared at $500^{\circ} \mathrm{C}$ has a higher content of iron (47\%) that may form hydroxides on exposure to air and water that could be responsible for increased adsorption of phenol. Functional groups present on the surface of the adsorbent may also participate in the adsorption process. While the difference between PNP adsorption by CAC-250 a CAC-500 was not as much as for phenol. The adsorption of PNP was very slightly enhanced by increasing $\mathrm{pH}$ of the solution. At lower $\mathrm{pH}$, PNP remains in the molecular form; therefore, there was no interaction between
PNP and the adsorbent, at alkaline $\mathrm{pH}$, the PNP become ionic and is attracted by the oppositely charged groups on the adsorbent. Along with the electrostatic attraction between adsorbent and adsorbate, the $\pi-\pi$ interaction between aromatic rings of phenols and adsorbents surface may also influence the adsorption of phenol and PNP. Adsorption of PNP and phenol was best fitted with the Freundlich isotherm model, except for adsorption of phenol by CAC-500 which indicates that the adsorption process is physical. A proposed mechanism for phenol and PNP adsorption is shown in Fig. 5.

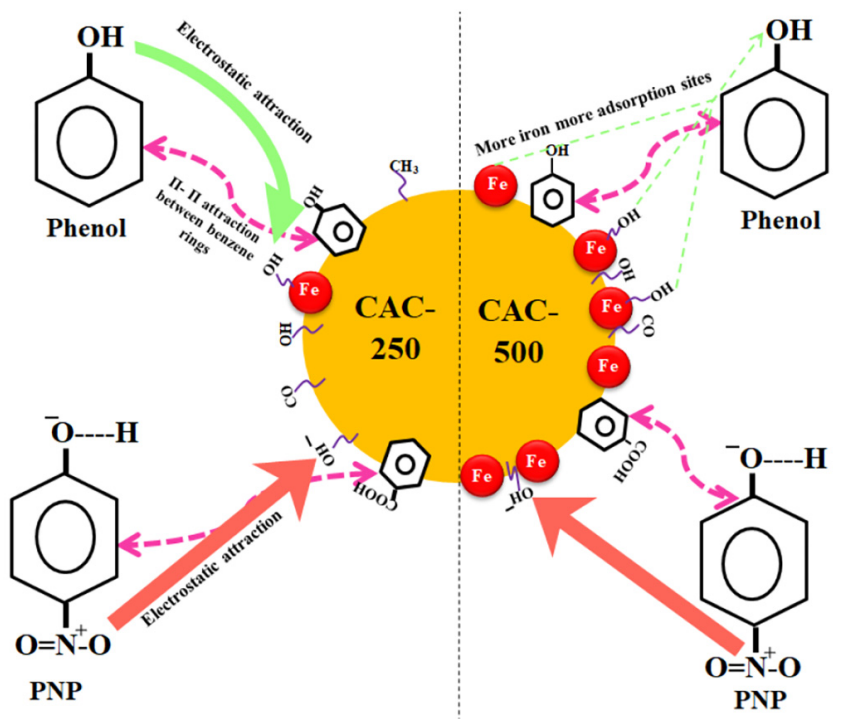

Fig. 5. Proposed mechanism for adsorption of phenol and PNP. 


\section{Conclusions}

The study compared the adsorption efficiencies of the prepared $\mathrm{AC}$ at two different temperatures $\left(250^{\circ} \mathrm{C}\right.$ and $\left.500^{\circ} \mathrm{C}\right)$. The study concludes that the adsorbent pyrolyzed at the higher temperature was found to be more efficient. The greater adsorption efficiency may result from more transformation of organic biomass into carbon at higher temperatures, and also more effective coating with iron that was evident in the elemental analysis of the adsorbents. The adsorption percentage of PNP by both the adsorbents was found to be higher, whereas phenol adsorption by CAC-250 was found very low, the maximum adsorption by CAC-250 was observed to be only $34.80 \%$ at minimum initial concentration of phenol. The adsorption process was examined by application of Langmuir, Freundlich and Temkin isotherms and adsorption kinetics. The adsorption data of phenol using CAC-250 was supported with Freundlich isotherm with $\mathrm{R}^{2}$ value 0.99 . The adsorption of PNP by both the adsorbents was supported by the Freundlich isotherm with the $\mathrm{R}^{2}$ value 0.98 and 0.94 for CAC-250 and CAC-500, respectively. The adsorption of both phenol and PNP was best fitted with the pseudo-second-order kinetics.

\section{Acknowledgments}

The authors are grateful to the Science and Engineering Research Board- Department of Science and Technology (SERB-DST) (project No. ECR/2016/001924) for providing financial support for this work. The authors are also thankful to the University Sophisticated Instrumentation Centre (USIC), BBAU, Lucknow for their kind help in the characterization of the materials.

\section{Author Contributions}

Ms. N.Y. and Mr. D.N.M. (M.Sc. Student) have done all the experiments. S.R. (Ph.D. student) has drafted the manuscript and Dr. J.S. (Assistant Professor) is the corresponding author who had supervised the work and made all the possible corrections in the manuscript.

\section{References}

1. Saleh TA, Adio SO, Asif M, Dafalla H. Statistical analysis of phenols adsorption on diethylenetriamine-modified activated carbon. J. Clean. Prod. 2018;182:960-968.

2. Rout K, Sahoo MK, Sahoo CR. Adsorption potential of BF slag and $\mathrm{BF}$ flue dust towards removal of aqueous phenol. Int. J. Adv. Res. Ideas Innov. Technol. 2018;4:116-121.

3. Öztürk H, Barışçı S, Turkay O, Veli S. Electrocatalytic degradation of phenol by the electrooxidation-electrocoagulation hybrid process: Kinetics and identification of degradation intermediates. J. Environ. Eng. 2019;145:04019014.

4. Tony MA, Mansour SA, Tayeb AM. Use of a fenton-like process based on nano-haematite to treat synthetic wastewater con- taminated by phenol: Process investigation and statistical optimization. Arab J. Sci. Eng. 2018;43:2227-2235.

5. Narkkun T, Boonying P, Yuenyao C, Amnuaypanich S. Green synthesis of porous polyvinyl alcohol membranes functionalized with L-arginine and their application in the removal of 4-nitrophenol from aqueous solution. J. Appl. Polym. Sci. 2019.

6. Abdel-Ghani NT, Rawash ESA, El-Chaghaby GA. Equilibrium and kinetic study for the adsorption of p-nitrophenol from wastewater using olive cake based activated carbon. GJESM 2016;2:11-18.

7. Sun M, Feng G, Zhang M, et al. Enhanced removal ability of phenol from aqueous solution using coal-based carbon membrane coupled with electrochemical oxidation process. Colloid. Surf. A Physicochem. Eng. Asp. 2018;540:186-193.

8. Ceylan Z, Mustafaoglu D, Malkoc E. Adsorption of phenol by MMT-CTAB and WPT-CTAB: Equilibrium, kinetic, and thermodynamic study. Part Sci. Technol. 2018;36:716-726.

9. Ayad MM, Amer WA, Zaghlol S. et al. Polypyrrole-coated cotton fabric decorated with silver nanoparticles for the catalytic removal of p-nitrophenol from water. Cellulose 2018;25:7393.

10. Álvarez-Torrellas S, Martin-Martinez M, Gomes HT, Ovejero G, García J. Enhancement of p-nitrophenol adsorption capacity through $\mathrm{N}_{2}$-thermal-based treatment of activated carbons. Appl. Surf. Sci. 2017;414:424-434.

11. Kowalczyk P, Deditius A, Ela WP, et al. Super-sieving effect in phenol adsorption from aqueous solutions on nanoporous carbon beads. Carbon 2018;135:12-20.

12. Lee C-G, Hong S-H, Hong S-G, Choi J-W, Park S-J. Production of biochar from food waste and its application for phenol removal from aqueous solution. Water Air Soil Pollut. 2019;230:70.

13. Cheng S, Zhang L, Xia H, Peng J. Characterization and adsorption properties of La and Fe modified activated carbon for dye wastewater treatment. Green Process Synth. 2017;6:487-498.

14. Tan Y, Sun Z, Meng H, et al. A new MOFs/polymer hybrid membrane: MIL-68(Al)/PVDF, fabrication and T application in high-efficient removal of p-nitrophenol and methylene blue. Sep. Purif. Technol. 2019;215:217-226.

15. Tian H, Hu Y, Xu X, et al. B. Enhanced wastewater treatment with high o-aminophenol concentration by two-stage MABR and its biodegradation mechanism. Bioresour. Technol. 2019; 289:121649.

16. Khan A, Chen Z, Zhao S, et al. Micro-aeration in anode chamber promotes p-nitrophenol degradation and electricity generation in microbial fuel cell, Bioresour. Technol. 2019;285:121291.

17. Massa A, Hernándeza S, Ansaloni S, Castellino M, Russo N, Fino D. Enhanced electrochemical oxidation of phenol over manganese oxides under mild wet air oxidation conditions. Electrochim. Acta. 2018;273:53-62.

18. Víctor-Ortega MD, Ochando-Pulido JM, Martínez-Férez A. Phenols removal from industrial effluents through novel polymeric resins: Kinetics and equilibrium studies. Sep. Purif. Technol. 2016;160:136-144.

19. Villegas LGC, Mashhadi N, Chen M, Mukherjee D, Taylor KE, Biswas N. A short review of techniques for phenol removal from wastewater. Curr. Pollut. Rep. 2016;2:157-167.

20. He D, Niu H, He S, Mao L, Cai Y, Liang Y. Strengthened fenton degradation of phenol catalyzed by core/shell Fe-Pd@C nano- 
composites derived from mechanochemically synthesized Fe-Metal organic frameworks. Water Res. 2019;162:151-160.

21. Guo X, He C, Sun X. Adsorption of phenol from aqueous solution by four types of modified attapulgites. Int. J. Environ. Sci. Technol. 2018;16:793-800.

22. Crini G, Lichtfouse E, Wilson $\mathrm{LD}$. Conventional and non-conventional adsorbents for wastewater treatment. Environ. Chem. Lett. 2018.

23. Hor KY, Mun J, Chee C, et al. Evaluation of physicochemical methods in enhancing the adsorption performance of natural zeolite as low-cost adsorbent of methylene blue dye from wastewater. 2016;118:197-209.

24. Dehghani MH, Sanaei D, Ali I, Bhatnagar A. Removal of chromium(VI) from aqueous solution using treated waste newspaper as a low-cost adsorbent: Kinetic modeling and isotherm studies. J. Mol. Liq. 2016;215:671-679.

25. Balarak D, Jaafari J, Hassani G, et al. The use of low-cost adsorbent (Canola residues) for the adsorption of methylene blue from aqueous solution: Isotherm, kinetic and thermodynamic studies. Colloid. Interf. Sci. Commun. 2015;7:16-19.

26. Abdelkreem M. Adsorption of phenol from industrial wastewater using olive mill waste. APCBEE Procedia 2013;5:349-357.

27. Liu B, Li Y, Gai X, Yang R, Mao J, Shan S. Exceptional adsorption of phenol and p-Nitrophenol from water on carbon materials prepared via hydrothermal carbonization of corncob residues. Biol. Resour. 2016;11:7566-7579.

28. Kumar NS, Asif M, Al-Hazzaa MI. Adsorptive removal of phenolic compounds from aqueous solutions using pine cone biomass: Kinetics and equilibrium studies. Environ. Sci. Pollut. Res. 2018;25:21949-21960.

29. Lunge S, Singh S, Sinha A. Magnetic ironoxide $\left(\mathrm{Fe}_{3} \mathrm{O}_{4}\right)$ nanoparticles from tea waste for arsenic removal. J. Magn. Magn. Mater. 2014;356:21-31.

30. Xu J-h, Gao N-y, Deng Y, Xia S-q. Nanoscale iron hydroxide-doped granular activated carbon (Fe-GAC) as a sorbent for perchlorate in water. Chem. Eng. J. 2013;222:520-526.

31. Cheng S, Zhang L, Xia H, Peng J. Characterization and adsorption properties of La and Fe modified activated carbon for dye wastewater treatment. Green Proc. Synth. 2017;6:487-498.

32. Singh J, Reddy KJ, Chang Y-Y, Kang S.-H, Yang J-K. A novel reutilization method for automobile shredder residue as an adsorbent for the removal of methylene blue: Mechanisms and heavy metal recovery using an ultrasonically assisted acid. Proc. Saf. Environ. Prot. 2016;99:88-97.

33. Asmaly H, Khan I, Abussaud B, et al. Adsorption of phenol on aluminum oxide impregnated fly ash. Desal. Water Treat. 2015;57:6801-6808.

34. Padmavathy KS, Madhu G, Haseena PV. A study on effects of $\mathrm{pH}$, adsorbent dosage, time, initial concentration and adsorption isotherm study for the removal of hexavalent chromium(Cr VI) from wastewater by magnetite nanoparticles. 2016;24: 585-594.

35. Mahmoodi NM, Hayati B, Arami M, Lan C. Adsorption of textile dyes on Pine Cone from colored wastewater: Kinetic, equilibrium and thermodynamic studies. Desalination 2011;268: 117-125.
36. Kilic M, Apaydin-Varol E, Putun AE. Adsorptive removal of phenol from aqueous solutions on activated carbon prepared from tobacco residues: Equilibrium, kinetics and thermodynamics. J. Hazard. Mater. 2011;189:397-403.

37. Hameed BH, Rahman AA. Removal of phenol from aqueous solutions by adsorption onto activated carbon prepared from biomass material. J. Hazard. Mater. 2008;160:576-581.

38. Yousef RI, El-Eswed B. The effect of $\mathrm{pH}$ on the adsorption of phenol and chlorophenols onto natural zeolites. Colloids Surf. A Physicochem. Eng. Aspects 2009;334:92-99.

39. Tang D, Zheng Z, Lin K, Luan J, Zhang J. Adsorption of p-nitrophenol from aqueous solutions onto activated carbon fiber. J. Hazard. Mater. 2007;143:49-56.

40. Haydar S, Ferro-Garciab MA, Rivera-Utrilla J, Joly JP. A dsorption of $p$-nitrophenol on an activated carbon with different oxidations. Carbon 2003;41:387-395.

41. Zhou Q, He HP, Zhu JX, Shen W, Ray L, Yuan FP. Mechanism of PNP adsorption from aqueous solution by $\mathrm{HDTMA}^{+}$-pillared montmorillonite-Implications for water purification. J. Hazard. Mater. 2008;154:1025-1032.

42. Gautam A, Rawat S, Verma L, et al. Green synthesis of iron nanoparticle from extract of waste tea: An application for phenol red removal from aqueous solution. Environ. Nanotechnol. Monit. Manage. 2018;10:377-387.

43. Hameed BH, Ahmad AA. Batch adsorption of methylene blue from aqueous solution by garlic peel, an agricultural waste biomass. J. Hazard. Mater. 2009;30;164:870-875.

44. Tang D, Zheng Z, Lin K, Luan J, Zhang J. Adsorption of p-nitrophenol from aqueous solutions onto activated carbon fiber. J. Hazard. Mater. 2007;143:49-56.

45. Weber WJ, Morris JC. Kinetics of adsorption on carbon from solution. J. Sanit. Eng. Div. 1963;89:31-60.

46. Wu F-C, Tseng R-L, Juang R-S. Initial behavior of intraparticle diffusion model used in the description of adsorption kinetics. Chem. Eng. J. 2009;153:1-8.

47. Kumar A, Jena HM. Preparation and characterization of high surface area activated carbon from Fox nut (Euryale ferox) shell by chemical activation with $\mathrm{H}_{3} \mathrm{PO}_{4}$. 2016;6:651-658.

48. Ma L, Chen Q, Zhu J, et al. Adsorption of phenol and Cu(II) onto cationic and zwitterionic surfactant modified montmorillonite in single and binary systems. Chem. Eng. J. 2016;283:880-888.

49. Abdel-Ghani NT, El-Chaghaby GA, Helal FS. Individual and competitive adsorption of phenol and nickel onto multiwalled carbon nanotubes. J. AdV. Res. 2015;6:405-415.

50. Ibrahim M, Siddique A, Verma L, Singh J, Koduru JR. Adsorptive removal of fluoride from aqueous solution by biogenic iron permeated activated carbon derived from sweet lime waste. Acta. Chim. Solv. 2019;66:123-136.

51. Mishra S, Yadav SS, Rawat S, Singh J, Koduru JR. Corn husk derived magnetized activated carbon for the removal of phenol $\mathrm{T}$ and para-nitrophenol from aqueous solution: Interaction mechanism, insights on adsorbent characteristics, and isothermal, kinetic and thermodynamic properties. J. Environ. Manage. 2019;246:362-373. 